\title{
Resultados a corto plazo de la artroplastia total de cadera con tallos femorales de fijación metafisaria de segunda generación
}

\author{
Eduardo J. Bochatey, ${ }^{*}$ Fernando A. Lopreite, ${ }^{* * *}$ \\ *Instituto de Tratamiento y Rehabilitación Articular, Ciudad Autónoma de Buenos Aires, Argentina \\ ${ }^{* * *}$ Servicio de Ortopedia y Traumatología, Hospital Británico de Buenos Aires, Ciudad Autónoma de Buenos Aires, Argentina
}

\begin{abstract}
RESUMEN
Introducción: Considerando la gran cantidad de artroplastias totales de cadera que se realizan hoy en pacientes jóvenes, es esencial comprender ampliamente la supervivencia de dichos implantes. La estabilidad del componente femoral y su resistencia al hundimiento son factores críticos para lograr una correcta osteointegración y el subsiguiente éxito clínico en la artroplastia total de cadera no cementada. Materiales y Métodos: Se llevó a cabo un estudio observacional, descriptivo, retrospectivo. Se analizó a todos los pacientes sometidos a un reemplazo total de cadera primario, por nuestro equipo, entre diciembre de 2017 y mayo de 2018. Se seleccionó a quienes se les habían colocado tallos de fijación metafisaria de segunda generación. Se compararon los valores obtenidos con nuestra base de datos de pacientes a los que se les colocaron tallos de primera generación. Resultados: Al evaluar las 82 artroplastias totales de cadera del grupo 1, contemplando el primer año de seguimiento posoperatorio, hallamos $2(2,44 \%)$ complicaciones registradas durante la cirugía (una fractura de calcar y una falsa vía generada mientras se trabajaba el canal femoral). Un paciente (2\%) de los 49 evaluados en el grupo 2 requirió una revisión del tallo femoral, por aflojamiento aséptico, a los 5 meses de la cirugía primaria. Conclusiones: Según nuestra experiencia, este tipo de implantes es conveniente para alcanzar resultados clínicos comparables con los obtenidos usando los de la generación anterior, pero disminuyendo el riesgo de complicaciones intraoperatorias durante su colocación. Es un método reproducible con una baja curva de aprendizaje en manos experimentadas.
\end{abstract}

Palabras clave: Artroplastia; prótesis no cementada; fijación metafisaria.

Nivel de Evidencia: IV

Short-term outcomes in total hip arthroplasties using second-generation metaphyseal fixation stems

\begin{abstract}
Introduction: The high number of total hip arthroplasties (THAs) currently being performed in young patients warrants a thorough understanding of THA survivorship. Femoral component stability and resistance to subsidence are critical factors to achieve correct osseointegration, and the subsequent clinical success, in uncemented THAs. Materials and Methods: We conducted an observational, descriptive, retrospective study on all patients who underwent primary total hip replacement performed by our surgical team between December 2017 and May 2018. We identified the patients who received second-generation metaphyseal fixation stems (Group 2). Group 2 results were then compared with our database of patients who received first-generation stems (Group 1). Results: Group 1: 82 THAs, of which, after a 1-year postoperative follow-up, 2 patients (2.44\%) had complications, which were noted during surgery (a calcar fracture and a false route caused while preparing the femoral canal). Group 2: 49 THAs, 1 patient (2\%) required femoral stem revision, due to aseptic loosening, 5 months after primary THA. Conclusions: In our experience, secondgeneration implants achieve clinical outcomes comparable to those obtained with first-generation implants, while also decreasing the risk of intraoperative complications associated with the placement of the implant. This method has shown to be reproducible and to have an easy learning curve for experienced surgeons.
\end{abstract}

Key words: Arthroplasty; uncemented prosthesis; metaphyseal fixation.

Level of Evidence: IV 


\section{INTRODUCCIÓN}

Se han logrado altas tasas de éxito en la artroplastia total de cadera (ATC) con el uso de los tallos femorales no cementados. ${ }^{1,2}$ Se estima que, para 2030, se incrementará significativamente la cantidad de ATC $^{3}$ y que, en la mayoría, se utilizarán implantes no cementados.

Teniendo en cuenta la gran cantidad de ATC que se realizan en pacientes jóvenes hoy en día, es esencial comprender ampliamente la supervivencia de dichos implantes. ${ }^{3,4}$

En la ATC no cementada, la estabilidad del componente femoral y su resistencia al hundimiento son factores críticos para lograr una correcta osteointegración y el subsiguiente éxito clínico. ${ }^{5,6}$

A fin de disminuir el micromovimiento y facilitar la osteointegración, es necesario el contacto íntimo entre el hueso cortical y el implante. La falta de dicho contacto puede provocar complicaciones clínicas, como hundimiento, aflojamiento aséptico o fracturas alrededor de la prótesis. ${ }^{7-9}$ Para obtener dicho contacto íntimo entre el hueso cortical y la prótesis, el diseño del tallo es un factor fundamental, aunque el diseño óptimo se desconoce y podría variar entre los pacientes.

En diciembre de 2017, nuestro equipo quirúrgico comenzó a utilizar tallos no cementados, de fijación metafisaria, de segunda generación (Accolade II; Stryker Orthopaedics, Mahwah, NJ, EE.UU.). Estos tallos difieren de los de primera generación en su diseño, tienen una curvatura medial variable específica para cada tamaño, que brinda un mayor contacto cortical, en un rango más amplio de morfologías femorales.

Estudios previos han demostrado que esta segunda generación de tallos no cementados, de fijación metafisaria, aumenta significativamente el press-fit femoral proximal y distal ( $82 \%$ vs. 54\% comparados con los de primera generación), alcanza un mejor llenado del canal en las secciones medial $(90,6 \%$ vs. $85,3 \%)$ y distal $(88,1 \%$ vs. $78,6 \%),{ }^{10}$ aumenta la fijación proximal y disminuye la distal, y reduce así el micromovimiento. ${ }^{11}$

Las mejoras de diseño en los tallos de segunda generación son: el aumento del radio de curvatura de la cara medial, específico para cada tamaño de canal femoral; el incremento del tamaño del tallo proporcional al crecimiento de dicho canal, la moderada reducción de la longitud del tallo $(\sim 15 \mathrm{~mm})$ y el relieve lateral distal. ${ }^{12}$

Estas modificaciones en su morfología no solo mejorarían el press-fit y la fijación, sino que también disminuirían el riesgo de fractura intraoperatoria, al adaptar el tamaño y la curvatura de su cara medial a la forma de cada tamaño femoral. En los tallos de primera generación, el tamaño varía solo a expensas de modificar su cara lateral, lo cual no permite controlar el impacto a nivel del calcar, tal como lo describen Colacchio y cols. ${ }^{12}$

El objetivo de este estudio fue comparar los resultados a corto plazo de ATC primarias entre pacientes a los que se les colocó un tallo de fijación metafisaria de segunda generación y aquellos con uno de primera generación. Se analizaron 1) la supervivencia de los implantes, 2) los resultados clínicos y los comunicados por el paciente, 3) los resultados radiográficos y (4) la incidencia de complicaciones intraoperatorias y posoperatorias inmediatas.

\section{MATERIALES Y MÉTODOS}

Se llevó a cabo un estudio observacional, descriptivo, retrospectivo. Se analizó a todos los pacientes operados de ATC primaria por nuestro equipo, entre diciembre de 2017 y mayo de 2018. Se seleccionó a los pacientes a los que se les habían colocado tallos de fijación metafisaria de segunda generación. Se compararon los valores obtenidos con nuestra base de datos de pacientes a quienes se les colocaron tallos de primera generación.

Para esta comparación, se dividió a los pacientes en dos grupos. El primer grupo incluyó a los pacientes con un tallo de primera generación y el segundo grupo, a aquellos con un tallo de segunda generación.

Los pacientes fueron evaluados rutinariamente, antes de la cirugía y se continuó con exámenes radiográficos posoperatorios a las 6 semanas, 3 y 6 meses, al año y una vez por año a partir de entonces, con el objetivo de detectar signos de osteólisis o aflojamiento. Se tomaron radiografías seriadas, de frente de ambas caderas y de perfil de la cadera reemplazada.

Para la evaluación funcional realizada en cada consulta programada, se tuvieron en cuenta la modificación del Harris Hip Score (respecto del registrado antes de la cirugía), el rango de movilidad, la discrepancia en la longitud de los miembros inferiores, las comorbilidades y las complicaciones.

Este estudio incluyó exclusivamente a pacientes sometidos a una ATC primaria con un tallo no cementado, de fijación metafisaria, ya sea de primera (grupo 1) o de segunda generación (grupo 2).

A todos se les colocaron tallos femorales Accolade (Stryker Orthopaedics, Mahwah, NJ, EE.UU.) de primera o de segunda generación, según el grupo de estudio. Dicho implante es fabricado a base de una aleación de titanio y tiene un diseño en cuña cónica. Presenta una cobertura de hidroxiapatita y su sección proximal está recubierta circunferencialmente de un aerosol de plasma (Figura 1). 


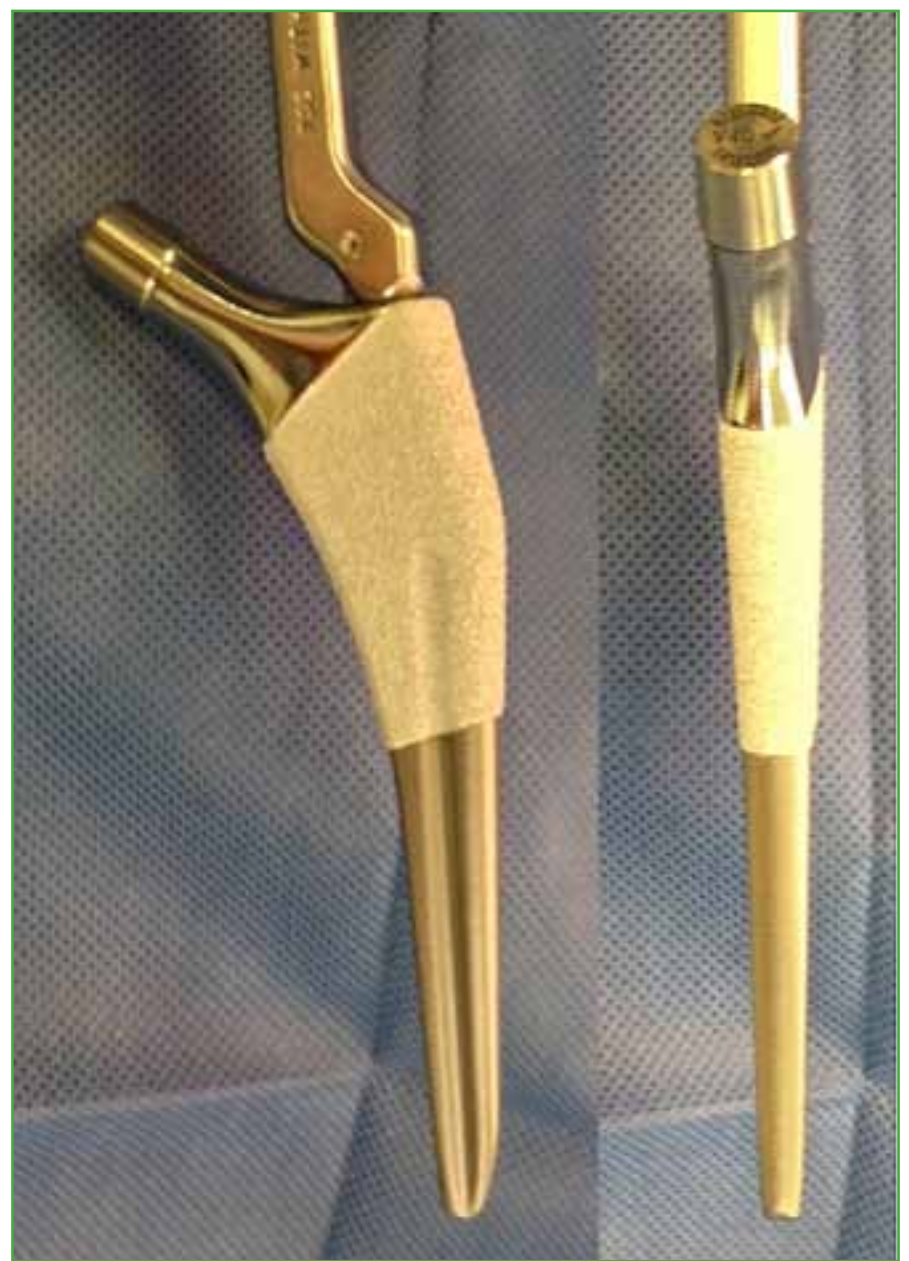

Figura 1. Tallo de segunda generación (Accolade II, Stryker Orthopaedics, Mahwah, NJ, EE.UU.).

El grupo 1, que recibió un tallo no cementado de fijación metafisaria de primera generación, Accolade TMZF (Stryker Orthopaedics, Mahwah, NJ, EE.UU.), incluyó 82 ATC en 75 pacientes (7 bilaterales): 41 hombres y 34 mujeres, con un promedio de edad de 53.5 años (rango 27-80). Cuarenta y seis de las 82 ATC fueron en caderas derechas $(56,1 \%)$ y $36(43,9 \%)$, izquierdas. Las causas de las ATC en este grupo fueron: coxartrosis (75 pacientes, 91,5\%), luxación congénita de cadera ( 2 pacientes, $2,44 \%$ ), conversiones de espaciador a ATC por artritis séptica (2 pacientes, $2,44 \%)$, secuela de artritis séptica ( 1 caso, $1,22 \%$ ) y necrosis avascular de la cabeza femoral (1 caso, $1,22 \%)$.

En el grupo 2, se estudiaron 50 ATC en 49 pacientes (una bilateral) realizadas con tallos de fijación metafisaria de segunda generación. De las 50 ATC, 24 se realizaron en 24 hombres (48\%) y 26, en 25 mujeres (52\%). El promedio de edad en el momento de la cirugía fue de 65 años (rango 35-79). Veintiséis procedimientos (52\%) fueron en caderas derechas y 24 (48\%), en caderas izquierdas. Las causas de las ATC primarias en este grupo fueron: coxartrosis (44 pacientes, $88 \%$ ), necrosis avascular de la cabeza femoral (3 pacientes, $6 \%$ ), secuelas de artritis séptica de cadera ( 1 caso, $2 \%$ ), luxación congénita de cadera ( 1 caso, $2 \%$ ) y fractura medial de cadera (1 caso, 2\%).

El seguimiento posoperatorio mínimo fue de un año, y se extendió a 18 meses en los pacientes operados en diciembre de 2017.

Todas las ATC estuvieron a cargo del mismo cirujano, se realizaron en cámara de flujo laminar, a través de un abordaje anterolateral de cadera modificado, con el paciente en posición supina, bajo anestesia raquídea, con profilaxis antibiótica de $1 \mathrm{~g}$ de cefalotina por vía intravenosa y administración de ácido tranexámico $(25 \mathrm{mg} / \mathrm{kg})$ durante la inducción anestésica. 
Como primer paso, se colocó el componente acetabular no cementado para luego realizar el trabajo del canal y la colocación del tallo femoral. Antes de la cirugía, se hizo la medición radiográfica del tamaño del tallo por utilizar y de la altura del corte en el cuello femoral, para evitar el sub o sobredimensionamiento del componente. Se trabajó en el canal femoral con raspas de tamaño ascendente. Finalmente, luego de pruebas de estabilidad y longitud, se impactó el tallo femoral definitivo.

\section{RESULTADOS}

$\mathrm{Al}$ evaluar al grupo 1, contemplando el primer año de seguimiento posoperatorio, se produjeron dos $(2,44 \%)$ complicaciones durante la cirugía. La primera fue una fractura de calcar, que se resolvió colocando una lazada de alambre en el mismo momento, evaluando la estabilidad inicial con la raspa de prueba y luego colocando el tallo definitivo (Figura 2). La segunda complicación fue una falsa vía generada mientras se trabajaba el canal femoral (Figura 3A), la cual se resolvió a las 24 h, en un segundo tiempo quirúrgico, con el correcto reposicionamiento del mismo componente primario (Figura 3B).

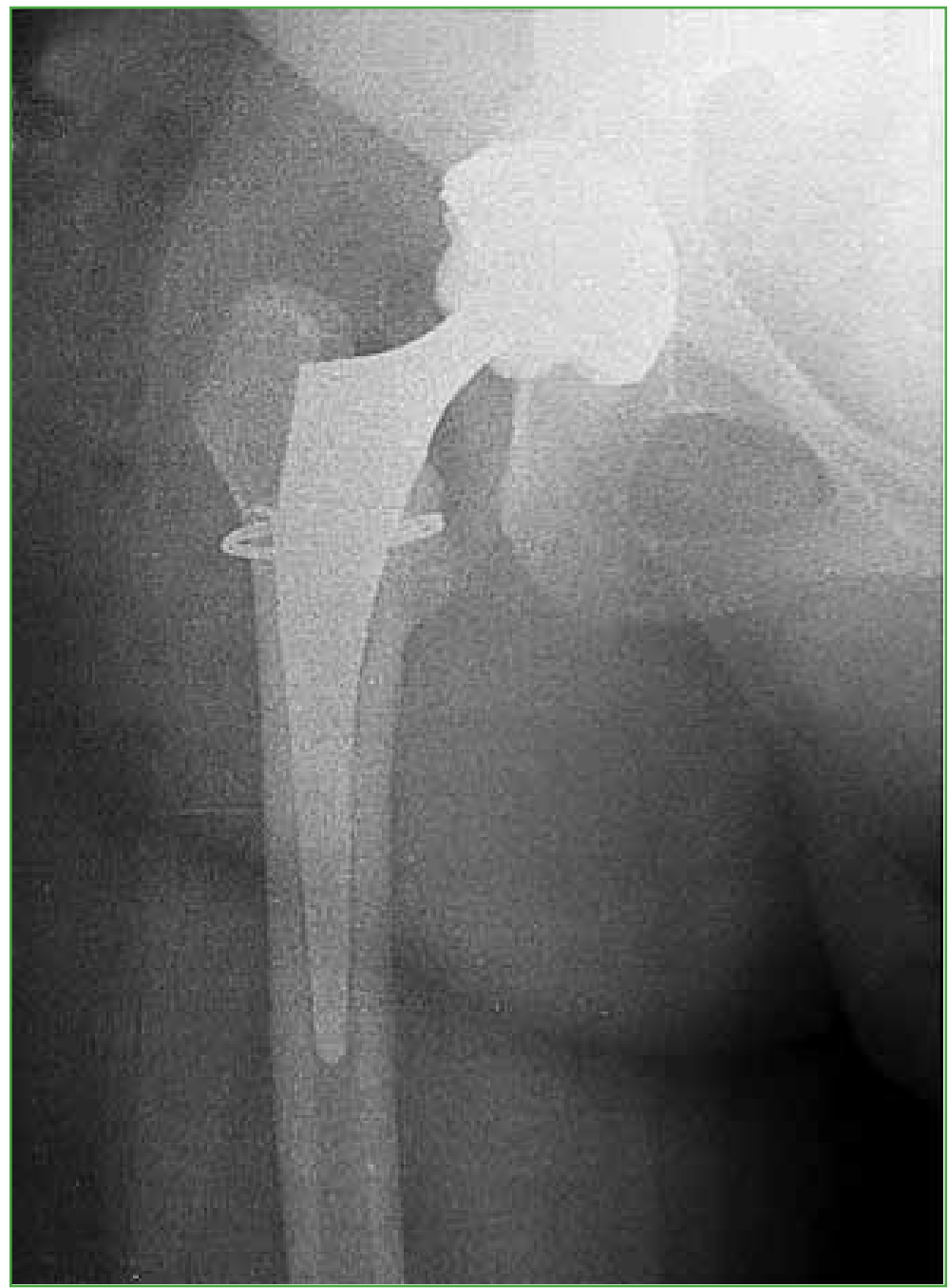

Figura 2. Tallo de primera generación con fractura de calcar intraoperatoria, que se resolvió con una lazada de alambre. 


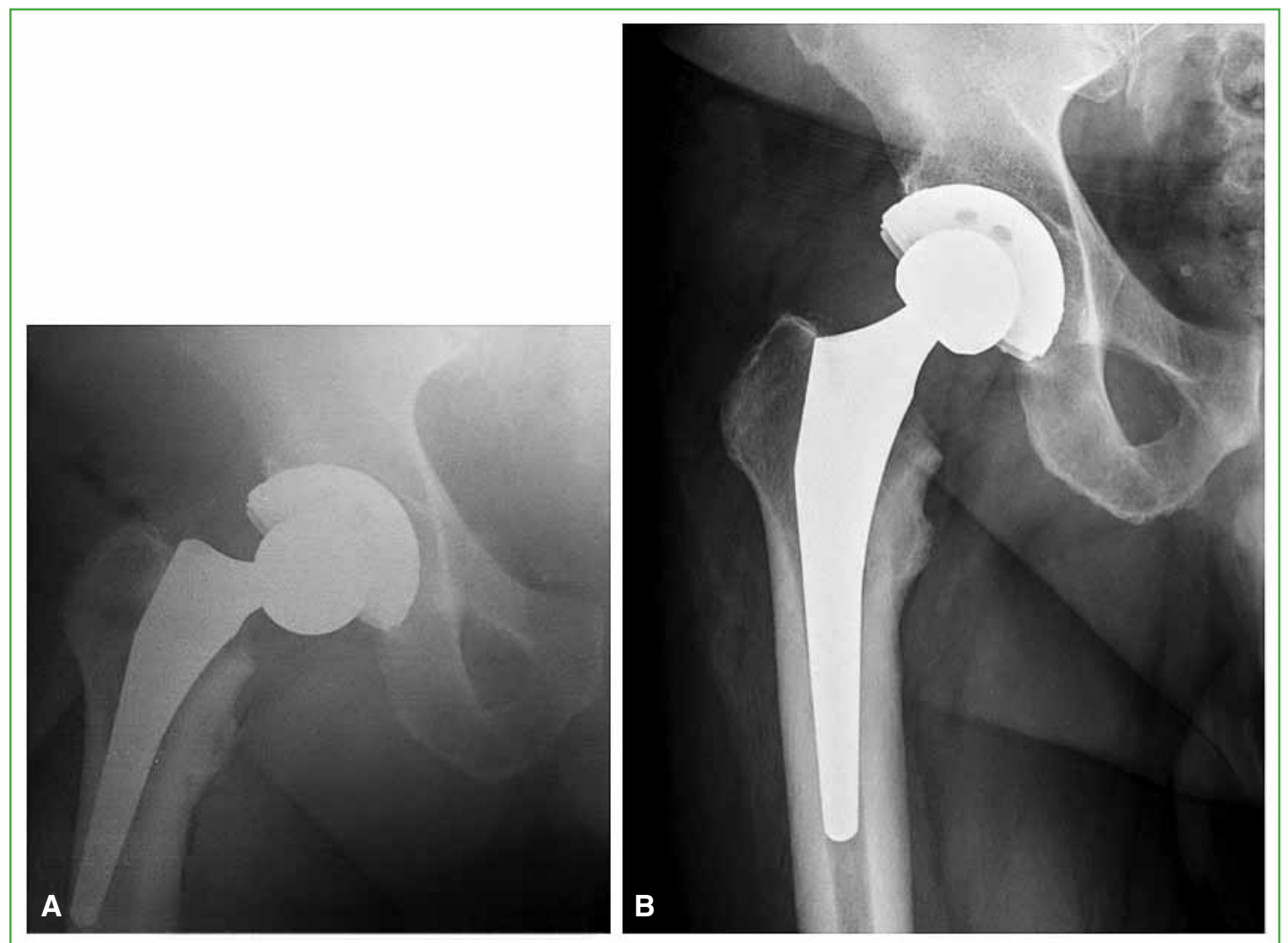

Figura 3. A. Control posoperatorio del tallo de primera generación con falsa vía femoral. B. Recolocación del tallo, luego de trabajar nuevamente el canal femoral.

En las restantes 80 ATC, se observó un correcto posicionamiento de los componentes, sin signos de osteólisis o desgaste en el control, al año de la cirugía. El rango de movilidad posoperatorio fue satisfactorio, sin registro de discrepancias significativas de la longitud de los miembros.

El Harris Hip Score preoperatorio promedio fue de 54 y ascendió a 95 en el control al año de la operación (Tabla).

Tabla. Comparación entre el grupo de tallos de primera generación (grupo 1) y el grupo de tallos de segunda generación (grupo 2)

\begin{tabular}{|l|c|c|}
\hline & Grupo 1 & Grupo 2 \\
\hline Sexo (femenino/masculino) & $34 / 41$ & $26 / 24$ \\
\hline Edad & $53.2(27-80)$ años & $65(35-79)$ años \\
\hline Lateralidad (derecha/izquierda) & $46(56,1 \%) / 36(43,9 \%)$ & $26(52 \%) / 24(48 \%)$ \\
\hline Harris Hip Score & $54-95$ & $56-95$ \\
\hline Complicaciones intraoperatorias & $2(2,44 \%)$ & $1(2 \%)$ \\
\hline
\end{tabular}


Un paciente (2\%) de los 49 evaluados del grupo 2 requirió una revisión del tallo femoral por aflojamiento aséptico, a los cinco meses de la ATC primaria (Figura 4A y B). Se reemplazó por un tallo cementado (Figura 4C).

El paciente sometido la revisión protésica por aflojamiento aséptico del tallo femoral consultó por dolor, a los cinco meses. En ese momento, se constataron signos radiográficos de aflojamiento y se descartó una infección. Entonces, se procedió a la revisión en un tiempo, en la que se constató la ausencia de osteointegración del implante. Al no encontrar errores en la colocación, ni fallas en el implante, la única causa atribuible al aflojamiento fue que la paciente fumaba 40 cigarrillos por día, que como sabemos, afecta la microcirculación endóstica y puede dificultar la osteointegración. En los 48 pacientes restantes, se confirmó un correcto posicionamiento de los componentes y no se observaron signos de osteólisis o aflojamiento en el último control radiográfico.

No hubo otras complicaciones ni comorbilidades. Tampoco se registraron diferencias significativas en la longitud de los miembros inferiores. El rango de movilidad posoperatorio fue satisfactorio, siempre la movilidad mejoró respecto de la registrada en la evaluación preoperatoria.

El aumento promedio del Harris Hip Score fue de 56 antes de la cirugía a 95 al año de la operación, en el grupo 2.

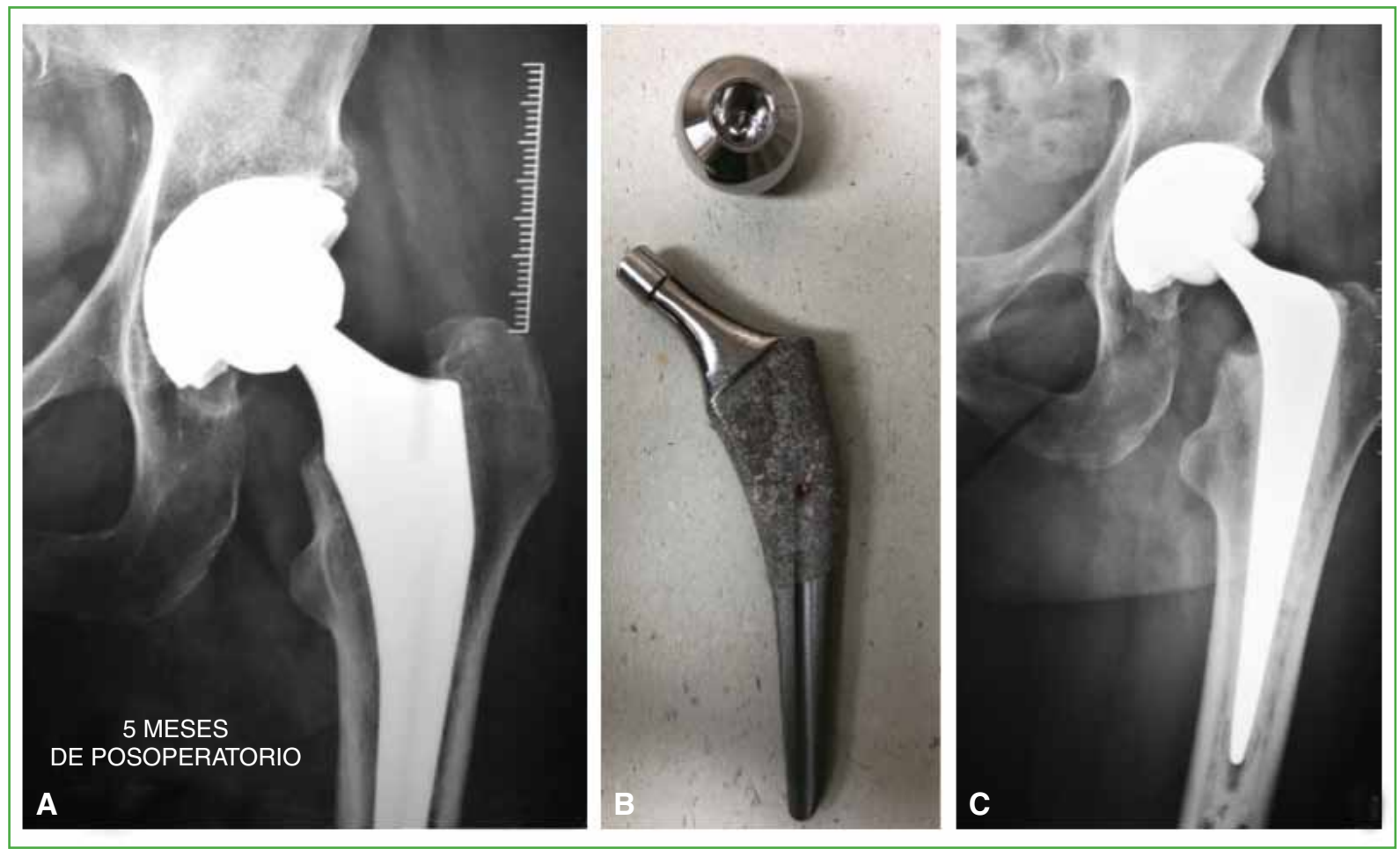

Figura 4. A. Demarcación en la cara medial del tallo de segunda generación. Signo de falla en la osteointegración.

B. Tallo extraído, sin signos de osteointegración. C. Control de la revisión con tallo cementado a los tres meses.

\section{DISCUSIÓN}

Se deben tener en cuenta varios aspectos para el diseño de un implante ideal de cadera, entre ellos, el material, el recubrimiento, los medios de fijación y la geometría. A medida que las características de los componentes femorales evolucionan, es esperable que los resultados y la satisfacción de los pacientes mejoren.

Estudios previos, como el de McLaughlin y cols., ya han demostrado excelentes resultados a largo plazo (seguimiento a 20 años) con los tallos no cementados, en cuña, cónicos. ${ }^{1}$

Los pacientes incluidos en este estudio fueron los primeros en recibir este implante por parte de nuestro equipo quirúrgico. Es común observar una curva de aprendizaje en la colocación de nuevos implantes, que puede generar una tasa más alta de errores iniciales, pero, según nuestra experiencia, los resultados indican una alta tasa de éxito 
a corto plazo, así como una mayor facilidad de adaptación para utilizarlos por parte del cirujano. Así lo describen Casper y cols., en su estudio sobre tallos de primera generación. ${ }^{3}$ Esta condición de reproducibilidad del método es una ventaja apreciable sobre otros sistemas.

Los tallos de segunda generación han tenido una supervivencia y una función excelentes a corto plazo. ${ }^{13}$

En nuestro estudio, hubo un caso de aflojamiento aséptico dentro del primer año con los tallos de segunda generación, y no se registraron episodios similares con los de primera. Esto puede atribuirse, como ya se mencionó, a la condición de fumadora de la paciente.

En contrapartida, no se produjeron complicaciones intraoperatorias con los tallos de segunda generación, pero sí con los de primera generación, una fractura de calcar y una falsa vía femoral, ambas mientras se trabajaba el canal $(2,44 \%)$. Esto concuerda con los resultados obtenidos por Colacchio y cols., quienes reportan un 3,27\% de fracturas de fémur intraoperatorias con tallos de primera generación y un $0,61 \%$ con los de segunda generación. ${ }^{12}$ Atribuimos esta diferencia en dicha complicación a una de las modificaciones más significativas en el diseño, como es el aumento del radio de curvatura de la cara medial, específico para cada tamaño de canal femoral. Esto permite un trabajo del canal que se ajuste más anatómicamente a él, evitando el impacto directo sobre el calcar, a medida que crece el tamaño de las raspas. Esto no es posible con los tallos de la generación anterior, ya que estos (al igual que las raspas correspondientes) modifican su tamaño solo a expensas de variar la cara lateral del tallo, su cara medial permanece con el mismo radio de curvatura.

Creemos que el análisis de esta complicación intraoperatoria es trascendental en el estudio de las complicaciones en un seguimiento a largo plazo, ya que, como lo describen Cooper y cols., este diseño particular de tallos de fijación metafisaria plantea un riesgo mayor de fracturas periprotésicas intraoperatorias, debido a la técnica de implantación y a la búsqueda de mejor press-fit, para alcanzar la estabilidad inmediata del componente, que permita una rehabilitación posoperatoria acelerada. ${ }^{14}$ Esta complicación sería menor en los tallos de fijación metafisaria de segunda generación por sus características del diseño geométrico.

Consideramos que las limitaciones de este estudio son su diseño de cohorte retrospectivo, el limitado número de pacientes y el corto tiempo de seguimiento posoperatorio.

Los puntos fuertes son que todos los pacientes incluidos fueron operados por el mismo cirujano y equipo quirúrgico, y que es el primer estudio en el país que evalúa los resultados con este tipo de implantes, convirtiéndolo en un estudio inédito a nivel nacional, esto nos permite comparar resultados con los obtenidos en otros países.

\section{CONCLUSIONES}

Este tipo de implantes es conveniente para lograr resultados clínicos comparables con los obtenidos con los de la generación anterior, pero con menor riesgo de complicaciones intraoperatorias durante su colocación. Es un método reproducible con una baja curva de aprendizaje en manos experimentadas.

Queda como trabajo pendiente continuar el seguimiento de estos implantes a mediano y largo plazo.

Conflicto de intereses: Los autores no declaran conflictos de intereses.

\section{BIBLIOGRAFÍA}

1. McLaughlin JR, Lee KR. Total hip arthroplasty with an uncemented femoral component. Excellent results at tenyear follow-up. J Bone Joint Surg Br 1997;79(6):900-7. https://doi.org/10.1302/0301-620x.79b6.7482

2. Parvizi J, Keisu KS, Hozack WJ, Sharkey PF, Rothman RH. Primary total hip arthroplasty with an uncemented femoral component: a long-term study of the taperloc stem. J Arthroplasty 2004;19(6):151-6. https://doi.org/10.1016/j.arth.2003.10.003

3. Casper DS, Kim GK, Restrepo C, Parvizi J, Rothman RH. Primary total hip arthroplasty with an uncemented femoral component. Five-to nine-year results. J Arthroplasty 2011;26(6):838-41.

https://doi.org/10.1016/j.arth.2011.02.010 
4. Hernández Cortes P, Nájera Sagastume OO, Mesa Ramos F, Pajares López M, Hernández Hernández MA. Hydroxyapatite-coated stems with metaphyseal and diaphyseal press-fit. Eleven-year follow-up results. Acta Orthop Belg 2002;68(1):24-32. PMID: 11915455

5. Streit MR, Innmann MM, Merle C, Bruckner T, Aldinger PR, Gotterbam T. Long-term (20- to 25-year) results of an uncemented tapered titanium femoral component and factors affecting survivorship. Clin Orthop Relat Res 2013;471(10):3262-9. https://doi.org/10.1007/s11999-013-3033-4

6. Pilliar RM, Lee JM, Maniatopoulos C. Observation on the effect of movement on bone ingrowth into poroussurfaced implants. Clin Orthop 1986;(208):108-13. PMID: 3720113

7. Capello WN, D’Antonio JA, Jaffe WL, Geesink RG, Manley MT Feinberg JR. Hydroxyapatite-coated femoral components: 15-year minimum followup. Clin Orthop 2006;453:75-80. https://doi.org/10.1097/01.blo.0000246534.44629.b2

8. Hwang K-T, Kim Y-H, Kim Y-S, Choi I-Y. Total hip arthroplasty using cementless grit-blasted femoral component: a minimum 10-year follow-up study. J Arthroplasty 2012;27(8):1554-61. https://doi.org/10.1016/j.arth.2012.02.005

9. Dalton J, Cook SD, Thomas KA, Kay JF. The effect of operative fit and hydroxyapatite coating on the mechanical and biological response to porous implants. J Bone Joint Surg Am 1995;77(1):97-110. https://doi.org/10.2106/00004623-199501000-00012

10. Faizan A, Wuestemann T, Nevelos J, Bastian AC, Collopy D. Development and verification of a cementless novel tapered wedge stem for total hip arthroplasty. J Arthroplasty 2014;30(2):235-40. https://doi.org/10.1016/j.arth.2014.09.023

11. Issa K, Pivec R, Wuestemann T, Tatevossian T, Nevelos J Mont MA. Radiographic fit and fill analysis of a new second-generation proximally coated cementless stem compared to its predicate design. J Arthroplasty 2014;29(1):192-8. https://doi.org/10.1016/j.arth.2013.04.029

12. Colacchio N, Robbins C, Aghazadeh M, Talmo C, Bono J. Total hip intraoperative femur fracture: does a tapered wedge design with a size specific medial curvature reduce the incidence of intraoperative femur fracture? J Arthroplasty 2017;32(10):3163-8. https://doi.org/10.1016/j.arth.2017.05.012

13. Kolisek FR, Chughtai M, Mistry JB, Elmallah RK, Jaggard C, Malkani AL, et al. Outcomes of second-generation tapered wedge femoral stem. Surg Technol Int 2016;28:275-9. PMID: 27042781

14. Cooper HJ, Rodríguez J. Early post-operative periprosthetic femur fracture in the presence of a non-cemented tapered wedge femoral stem. HSS J 2010;6(2):150-4. https://doi.org/10.1007/s11420-010-9161-4 